\title{
Requerimientos del Mercado laboral y publicidad de diversas IES. Caso Carrera de Mercadotecnia, Guayaquil
}

\section{Requirements of the labor market and advertising of various IES. Case: Marketing career, Guayaquil}

\author{
Mgtr. Guido Mantilla Buenaño ${ }^{1}$ \\ gmantillab@ulvr.edu.ec \\ https://orcid.org/0000-0002-5598-8330 \\ Mgrt. Félix David Freire Sierra ${ }^{2}$ \\ ffreires@ulvr.edu.ec \\ https://orcid.org/0000-0002-7393-3229 \\ Mgtr. Marisol Jacqueline Mercedes Idrovo Avecillas 3 \\ midrovoa@ulvr.edu.ec \\ https://orcid.org/0000-0002-0176-9917 \\ Mgtr. Martha Hernández Armendariz ${ }^{4}$ \\ mhernandeza@ulvr.edu.ec \\ https://orcid.org/0000-0002-9518-9031
}

Recibido: 28/9/2021, Aceptado: 28/12/2021

\begin{abstract}
RESUMEN
El presente estudio sintetiza parte de los resultados de una investigación sobre "Oferta y demanda académica de la carrera de Mercadotecnia en diversas Instituciones de Educación Superior". El proyecto investigación fue respaldado por la Universidad Laica Vicente Rocafuerte de Guayaquil. Durante su ejecución, se realizaron entrevistas a empresarios y Gerentes de Marketing de 10 PYMES. El cuestionario consideró 5 preguntas sobre los requerimientos del mercado empresarial. Se utilizó la observación durante 45 días encontrándose 16 instituciones que ofertaban sus productos académicos de variadas formas. La ficha de observación comprendía 6 campos y un check list de 18 items. Como requerimientos del mercado destacan: el Marketing debe ser tratado en la academia con un enfoque holístico y estratégico; el mercadólogo requiere destreza en el manejo de redes sociales así como contar con capacidad de aprendizaje, adaptación, destreza oral, escrita y numérica; con respecto a la publicidad se observó que dicha oferta es bastante agresiva. Los lugares de publicación son ingeniosos. A nivel digital son utilizadas las redes sociales; de manera física, se incluyen: aeropuerto, cines, baños de los cines, pantallas gigantes, displays de centros comerciales, buses, taxis y los medios de comunicación habituales: TV, radio y prensa escrita.
\end{abstract}

Palabras clave: Mercado laboral, promoción publicitaria, IES, marketing digital, redes sociales

\footnotetext{
${ }^{1}$ Universidad Laica Vicente Rocafuerte, Ecuador

2 Universidad Laica Vicente Rocafuerte, Ecuador

3 Universidad Laica Vicente Rocafuerte, Ecuador

${ }^{4}$ Universidad Laica Vicente Rocafuerte, Ecuador
} 


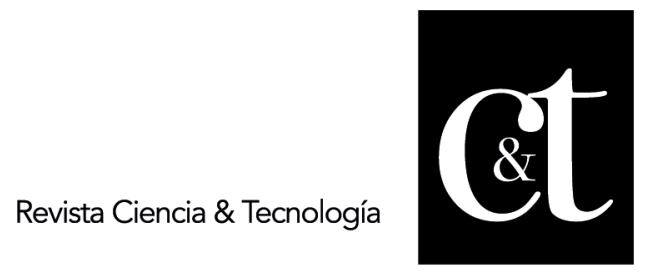

No. 33, 31 de enero de 2022

ISSN impreso: 1390 - 6321

ISSN online: 2661 - 6734

\begin{abstract}
The present study synthesizes part of the results of an investigation on "Academic supply and demand of the Marketing career in various Higher Education Institutions". The research project was supported by the Vicente Rocafuerte Secular University of Guayaquil. During its execution, interviews were conducted with entrepreneurs and Marketing Managers of 10 SMEs. The questionnaire considered 5 questions about the requirements of the business market. Observation was used for 45 days, finding 16 institutions that offered their academic products in various ways. The observation file comprised 6 fields and a check list of 18 items. As market requirements stand out: Marketing must be treated in the academy with a holistic and strategic approach; the marketer requires skill in the management of social networks as well as having the ability to learn, adapt, oral, written and numerical skills; regarding advertising, it was observed that said offer is quite aggressive. Publishing places are nifty. At the digital level, social networks are used; In a physical way, they include: airport, cinemas, movie theaters bathrooms, giant screens, displays in shopping centers, buses, taxis and the usual means of communication: TV, radio and written press.
\end{abstract}

Keywords: Labor market, advertising promotion, IES, digital marketing, social networks

\title{
Introducción
}

"El entorno de la enseñanza superior y de la sociedad en general está sufriendo hasta hoy cambios de gran trascendencia; los rankings internacionales de calidad son una manifestación del nuevo contexto de competitividad de las universidades" (Duro \& Gilart, 2016, p. 166). Las universidades ofrecen muchas carreras a elegir a distintos niveles y en variadas modalidades. En Ecuador, la Secretaría de Educación, Ciencia, Tecnología e Innovación (2021) detalló que sólo en el primer período académico del presente año, un total de 94349 cupos están disponibles en 218 instituciones de educación superior. Por otra parte es imprescindible que esta oferta tan diversa corresponda plenamente con los requerimientos del mercado laboral cada vez más dinámico y competitivo.

Las universidades deben adaptarse y responder a nuevas demandas y requerimientos del entorno, competir con otras instituciones, extender su campo de acción y demostrar eficiencia (Duro \& Gilart, 2016), puesto que "la educación es también un servicio, buscado por múltiples consumidores, que requiere una gestión bastante cuidada y rigorosa" (Caetano, 2003, p.13)

Con estos antecedentes a finales del 2019, en la Universidad Laica Vicente Rocafuerte de Guayaquil surgió la inquietud de plantear un proyecto de investigación sobre "Oferta y demanda académica de la carrera de Mercadotecnia en diversas Instituciones de Educación Superior". El proyecto fue aprobado por el Departamento de Investigación Científica, Tecnológica e Innovación -DICTI- de la universidad, comenzó su ejecución a inicios del 2020 y se tomó un año. Formamos parte de la investigación un grupo de docentes investigadores. 


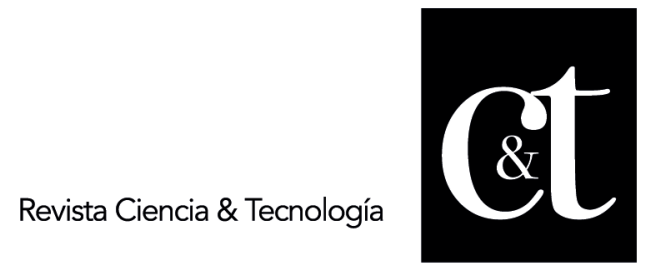

No. 33, 31 de enero de 2022

ISSN impreso: 1390 - 6321

ISSN online: 2661 - 6734

El proyecto se desarrolla en vista de la importancia que ha cobrado el Marketing y específicamente el Marketing digital a raíz de la pandemia mundial y uno de los principales objetivos es poner a consideración de la academia y del mercado documentación que exponga sobre las necesidades del mercado sobre los profesionales de Mercadotecnia en pos de que se desarrollen proyectos educativos que las satisfagan y que éstos sean promocionados éticamente para lograr el bienestar.

En el presente estudio compartimos al lector parte de los resultados de la investigación realizada en la Universidad Laica Vicente Rocafuerte, pues si bien es cierto la demanda abarca tanto los requerimientos de los bachilleres también comprende los requerimientos del mercado laboral. Se conoce de antemano que el trabajo híbrido, inclusión, adaptación al cambio y liderazgo son algunas de las tendencias que estarán presentes en el mundo laboral durante el desarrollo del año (Grupo Adecco, 2021).

Desde el punto de vista de la oferta académica se podría considerar: el grado de satisfacción de los estudiantes de la carrera de mercadotecnia, las carreras y diversos niveles - modalidades, la publicidad utilizada por las Instituciones de Educación Superior (IES).

Cabe destacar que en esta contribución se presentan los requerimientos del mercado laboral y la oferta publicitaria de diversas IES de la ciudad de Guayaquil, que en cierta forma es parte de la cultura organizacional y de la inteligencia competitiva de las mismas.

\section{Contribuciones teóricas}

\section{La formación universitaria en la carrera de Marketing.}

Definitivamente la educación universitaria en cualquiera de sus niveles es una tarea difícil y que acorde a los requerimientos del mercado siempre debe someterse a mejora continua; pero, para quienes nos apasiona la cátedra es una motivación, una responsabilidad y una oportunidad de respaldar el cambio y progreso humano.

Hacer observación y seguimiento de la realidad educativa es un proceso complejo. En vista de la dinámica de la educación superior, exige la exploración constante del contexto e individuos. Este proceso responde a estrategias definidas para tal efecto; una de ellas es la sistematización de experiencias (Barbosa et al, 2015).

Se tiene como meta formar profesionales con sólidos conocimientos en Marketing, capacidad para adaptarse fácilmente a un entorno, liderar e innovar la definición y diseño de productos, servicios y marcas a nivel estratégico y por supuesto también a una persona capaz de generar y llevar adelante emprendimientos.

En España, los nuevos profesionales no sólo deberán tener conocimientos de Marketing y Comunicación sino también de programación y gestión de datos. 


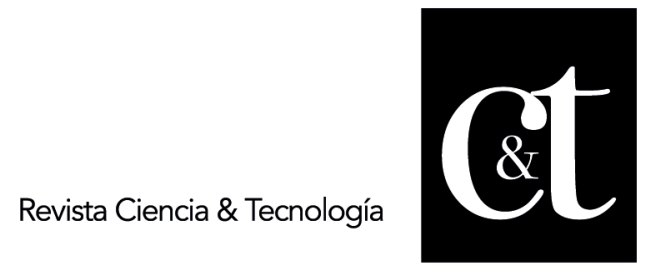

No. 33, 31 de enero de 2022

ISSN impreso: 1390 - 6321

ISSN online: 2661 - 6734

Las empresas de marketing/publicidad y agencias de medios necesitan nuevos perfiles orientados a la informática, las matemáticas y la estadística, pero sobre todo se busca un profesional de la comunicación digital que sea un híbrido entre analista de

datos y estratega, lo que se reúne en nuevos perfiles vinculados a las ciencias de la información, tales como media trader, programmatic media planner, data scientist y data intelligence (Carrillo \& Rodriguez, 2018).

En la actualidad, la inestabilidad en el mercado de trabajo hace que sea necesario que los jóvenes planifiquen su carrera y al buscar formación profesional tengan algunas motivaciones como: corta duración de la carrera, la realización de prácticas en la empresa, el aprendizaje lúdico que le brinde el centro de estudios, encontrar trabajo con facilidad al terminar los estudios y el tener orientación profesional. (Núñez, 2010).

\section{Tecnología y educación virtual}

Las nuevas tecnologías han impactado al mundo actual. En educación se debe utilizar la tecnología con todos sus avances y de la manera más eficaz, de tal manera que no importe la distancia y el tiempo. Ha existido una revolución para la enseñanza no presencial, donde los pedagogos deben desaprender métodos tradicionales. Ningún medio es bueno o malo en sí, siempre va a depender de quién y cómo se utilice. (Begoña, 2004)

Las TICs han permitido la oportunidad de presentar, on line más y mejores estudios para las distintas clases sociales; ofrecer mayor apertura y modificación de los estudios. (Rosario, 2006).

\section{Publicidad de IES}

La dura competencia por estudiantes, recursos y prestigio influye en las IES a buscar las maneras más idóneas y/u óptimas de diferenciarse y establecer alguna ventaja competitiva; existe incluso presión por darse a conocer, captar demanda y fidelizar a los alumnos.

Estudios acerca de la cultura de masas han contribuido a explicar diversos patrones de consumo que han impactado en la manera en que se desarrolla la publicidad la que, en ocasiones, puede estar cargada de simbolismo y representaciones que son justamente el fundamento del consumo de las personas (Zapata \& Tejeda, 2016, p.208)

La mercantilización de la educación ha traído diversas transformaciones y si bien la publicidad predomina en diferentes medios y lugares físicos y digitales, la promoción de la información y de la transparencia, son claves para sentar las bases de confianza en el mercado. En Chile por ejemplo, se planteó este control desde hace más de una década. En Ecuador, la Ley Orgánica de Defensa al Consumidor al 2021 menciona sobre publicidad engañosa pero se requiere un control se promueva. (Zapata \& Tejada, 2016).

Con la publicidad prácticamente no sólo se persigue dar a conocer el producto o el servicio que se oferta sino que los consumidores permanezcan por largo tiempo fidelizados. 


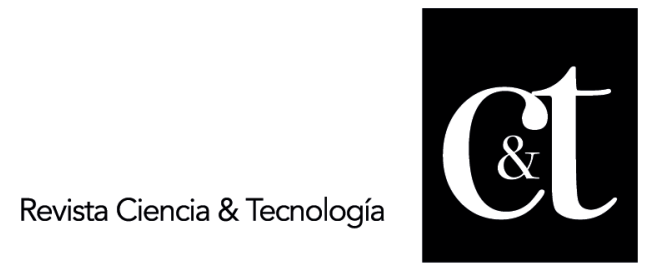

No. 33, 31 de enero de 2022

ISSN impreso: 1390 - 6321

ISSN online: 2661 - 6734

Segura et al (2019) en una investigación titulado "La evaluación de la publicidad en la prensa escrita de Ecuador, un estudio longitudinal" destacan el predominio de contenidos promocionales de universidades privadas que se dirigen a estudiantes potenciales y el valor que persiste de la prensa, a pesar del auge de la publicidad on line.

\section{Metodología}

El tipo de investigación realizado para la presente publicación es descriptivo y exploratorio, de diseño no experimental y de enfoque cualitativo.

La investigación descriptiva se refiere a la caracterización de un hecho, fenómeno, individuo o grupo con el fin de establecer su estructura o comportamiento (Arias, 2012).

Realizamos una revisión de literatura a nivel local y encontramos que existen pocas investigaciones como la propuesta, por tanto consideramos a esta obra de carácter exploratorio.

"La investigación es de naturaleza exploratoria, lo que significa que se trabaja en pequeña escala para identificar mediante datos empíricos los aspectos fundamentales que permitan brindar una visión general, de tipo aproximativo, de un determinado fenómeno o realidad" (Rodríguez et al, 2017, p.133)

El enfoque cualitativo de investigación utiliza la recolección y análisis de datos para afinar las preguntas de investigación o revelar nuevas interrogantes en el proceso de interpretación (Hernández et al, 2014). En el diseño no experimental no se analiza el efecto producido por la manipulación de una o más variables independientes sobre una o más variables dependientes (Bernal, 2010).

\section{Requerimientos del mercado laboral}

Para analizar los requerimientos del mercado laboral sobre profesionales de Mercadotecnia se utilizó como técnica de investigación la entrevista. El estudio fue transversal. La entrevista es el medio que permite la obtención de información de fuente primaria, de manera amplia y abierta. Se considera necesario que el entrevistador tenga definido los aspectos relevantes sobre los que se pretende obtener información (Fresno, 2019).

Por medio del método hermenéutico hemos orientado a generar interpretaciones sintéticas y realizar análisis sobre las opiniones vertidas en entrevistas a empresarios y directivos de empresas sobre los requerimientos del mercado laboral sobre los profesionales de Marketing y la formación que la academia ofrece en esta carrera.

Cárcamo (2005) sostiene que aunque la hermenéutica ha sido utilizada sobre todo por la necesidad de reconocer al historicismo como elemento fundamental para el "desarrollo" de las sociedades. Añade también que utilizar la hermenéutica implica la comprensión de los textos a partir del ejercicio interpretativo intencional y contextual.

El instrumento de investigación en este caso fue un cuestionario de 5 preguntas que se aplicó a 10 empresarios y/o Gerentes de Marketing de algunas PYMES, expertos que fueron elegidos a conveniencia. 


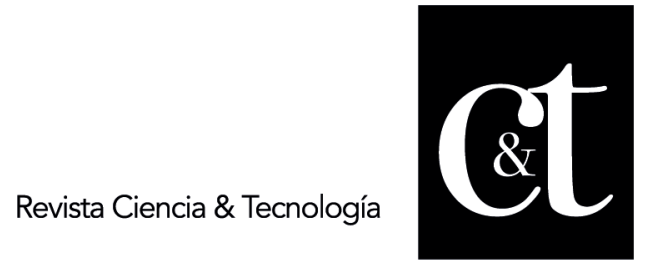

No. 33, 31 de enero de 2022

ISSN impreso: 1390 - 6321

ISSN online: 2661 - 6734

Los entrevistados eran propietarios o desempeñaban cargos estratégicos importantes en distintas PYMES del país, las cuales son:

- UniLimpio, proveedor internacional de productos y servicios limpieza a nivel industrial en Ecuador y Perú;

- UDLA, Universidad de las Américas;

- Mariscos Flipper, comercializadora y distribuidora de pescados frescos congelados y empacados al vacío a nivel nacional;

- Director Comercial de Elementia - Eternit Ecuatoriana, comercializadora y productora de materiales de construcción con más de 200 puntos de distribución en el país;

- Proaño Representaciones S.A., Empresa de Importación y Exportación con sede en Quito y de PremiunCorp, comercializadora de equipos de bio-seguridad, protección personal y salud ocupacional; .

- Solís Pharma, comercializadora de productos farmacéuticos a nivel nacional, entre las principales.

El trabajo de campo fue realizado enteramente en línea a través de la plataforma Google Meet y el contacto con los 10 entrevistados fue mediante email y línea telefónica. Fue fortuna concretar las entrevistas con algunos de los entrevistados si se considera sus ocupaciones habituales al gestionar empresas con 10 o más empleados.

\section{Publicidad de diversas IES}

Para analizar la publicidad de las IES de la ciudad se utilizó como técnica de investigación la observación; el estudio fue longitudinal.

La observación conduce a la necesidad de la sistematización de los datos y se refiere a la percepción visual utilizada registrando posibles características relevantes de un fenómeno (Gómez, 2012).

La ficha de observación fue diseñada con 6 campos junto con un check list de 18 items. Se aprovechó la matriculación a un nuevo semestre que se dio en septiembre de 2020, días en los cuales el auge de propagandas fue relevante. El trabajo de campo fue físico y digital y se dividió entre los docentes participantes quienes observamos la publicidad colocada por las IES en los principales sectores de la ciudad lo cual incluía avenidas principales y más transitadas así como los principales centros comerciales. Las visitas digitales incluyeron la observación de los principales sitios que brindan servicio de correo electrónico gratuito, las populares redes sociales (en especial: Facebook, Instagram, Twitter y Tik Tok) las búsquedas en Google y Yahoo, entre otras.

\section{Resultados y discusión}

Requerimientos del mercado laboral

Como expusimos, esta primera parte de la investigación se realizó utilizando la técnica de la entrevista. A continuación se presentan las preguntas realizadas con su respectivo análisis 


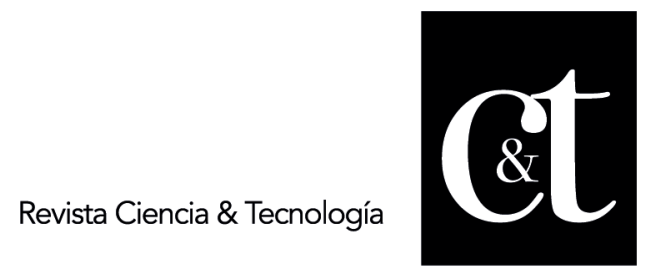

No. 33, 31 de enero de 2022

ISSN impreso: 1390 - 6321

ISSN online: 2661 - 6734

\section{Pregunta 1}

Qué competencias, destrezas, conocimientos requiere una persona que trabaje en el Departamento de Marketing?

La mayoría de los entrevistados coinciden en que se requieren varias habilidades y destrezas. Entre las competencias se mencionan: adaptabilidad, capacidad de aprendizaje, análisis numérico, comunicación escrita, trabajo en equipo, iniciativa. Entre las destrezas requeridas se solicitan destrezas orales, escritas y numéricas manejo de redes sociales.

Se solicita también sólidos conocimientos de gestión en Marketing digital, Relaciones públicas, redes sociales, Planeación estratégica, entre otros.

Hay dos cosas importantes. La primera: que tienen que tener la capacidad de hacer un plan estratégico, donde no sólo vincule al tema de comunicación, sino las ventas. Que contemple la perspectiva interna, la perspectiva de cliente, la perspectiva de aprendizaje; es decir, que tenga la capacidad de poder diseñar un plan estratégico que englobe muchas cosas, incluido la fuerza de Ventas. En segundo lugar: que ese plan estratégico lo pueda convertir en ventas... (AC, Eternit).

Es fundamental: la creatividad, la comunicación y la adaptación, el manejo de la tecnología, el resto lo va adquiriendo. Pero el chico no tiene bases, ni conocimientos consistentes de cómo opera una empresa, no tendrá éxito... Es muy valiosa la facilidad que le de la universidad con respecto a prácticas preprofesionales. (LP, Maricos Flipper).

... indudablemente que las competencias o destrezas, orales, escritas y numéricas son de gran relevancia. Hablando de la actividad turística, el tiempo de respuesta a un cliente, debe ser el menor posible. Esto amerita también un gran discernimiento de problema, una ágil toma de acciones y además saber gestionar. Es importantísimo que las generaciones actuales estén siendo educadas para trabajar bajo presión, tomar decisiones, resolver problemas.... En el tema turístico todo está volcándose muchísimo a todo el mundo digital. La utilización de herramientas digitales son de consumo mundial. (IJ, HDP Agencia mayorista de turismo).

Lo anteriormente expuesto se respalda con las conclusiones emitidas por García \& Echegaray (2018) en una investigación sobre las competencias y habilidades del profesional del Marketing, en la cual encontraron que como competencias concretas se necesitan el trabajo en equipo, presentaciones en público, relaciones cooperativas, comunicación interpersonal y negociación. Estas son habilidades detectadas que siguen siendo requeridas por la empresa, sin que pueda observarse ningún cambio en el medio plazo. Con respecto al Marketing digital se debe tratar aquellas habilidades relacionadas con la formulación de estrategias de CRM, incluyendo el manejo de bases de datos y Data Warehouse. En definitiva, se requieren también en todo un conjunto de habilidades más intangibles, mucho menos medibles, y cuya demanda será creciente en los próximos años.

Revista científica Ciencia y Tecnología Vol 22 No 33 págs. 21-36 http://cienciaytecnologia.uteg.edu.ec 


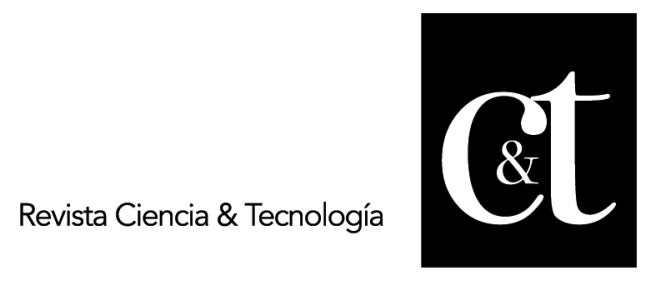

No. 33, 31 de enero de 2022

ISSN impreso: 1390 - 6321

ISSN online: 2661 - 6734

\section{Pregunta 2 \\ Cuáles son las especialidades de Marketing más demandadas por el mercado empresarial?}

Al responder a esta pregunta, primero surgió una aclaración importante sobre la diferencia entre Marketing y Ventas como tales; así como sobre la importancia del Marketing en todas las empresas. Se recalca también la necesidad de que el mercadólogo esté empoderado de su rol en la organización.

Existe un problema de comprensión sobre la carrera en sí, siempre se piensa que la persona que estudia Marketing, va a hacer ventas y eso es errado,... pero como mercadólogo me considero que soy de gran apoyo para la gestión de ventas a la hora de vender, justamente por las capacidades orales o por las capacidades de análisis numéricas que tengo. (AZ, UDLA). Es que el Marketing es para mí es la esencia misma de la organización. Con el alto nivel de competencia de una persona, Steve Jobs dice que no es suficiente, que ahora hay que contratar el corazón de la persona, empoderarlos, hacer que formen parte de este proyecto, de este sueño, de esta visión. Hoy yo tengo el pleno convencimiento de que las organizaciones revolucionarias, las organizaciones de proyección internacional y mundial tienen que tener una cabeza estratégica, una cabeza de Marketing, no una cabeza administrativa, no una cabeza de producción, no, todo eso ya es inherente en el proceso de una organización, pero la cabeza estratégica, la cabeza de Marketing es infalible. (EF, UniLimpio).

Respondiendo específicamente a la pregunta, los empresarios y directivos de Marketing hacen énfasis en que el mercado laboral es bastante demandante, que la tecnología juega un papel primordial y que se require un dominio del Marketing digital, e-commerce, e-business, community management y afines a la par con la parte estratégica para que los negocios tengan permanencia, y éxito.

La pregunta que ustedes plantean no serían especialidades, sino subespecialidades, como estaríamos diciendo que hay menciones, menciones en redes sociales, menciones en Diseño Gráfico, porque en ocasiones nos encontramos que busco un egresado de Marketing, o busco un profesional en Mercadeo, pero que sepa utilizar programas de Ilustración, programas de Diseño y que aparte de eso también haga manejo de redes sociales, ¿qué estamos pidiendo?, estamos pidiendo una persona que vaya a hacer o vaya a cumplir todas las funciones, que deben realizar diferentes personas en una empresa.( $A Z$, $U D L A$ )

Yo veo que el Marketing está en todo, pero obviamente tienes que especializarte y empezar a desarrollar en base a cada área en la que tú te especialices o te dirijas. Charles Darwin dice que no sobrevive el más fuerte, ni el más inteligente, sino el que mejor se adapte a las circunstancias actuales, ese cambio de estado nos está llevando a una nueva normalidad. La era digital que venía a un paso rápido, de repente se volvió una necesidad imperativa, entonces si ustedes son de Marketing y están viendo las oportunidades, el mundo corrió de repente por otro camino que se llama la era digital, te guste o no te guste tendrás que embarcarte caso contrario corres el riesgo de desaparecer. 


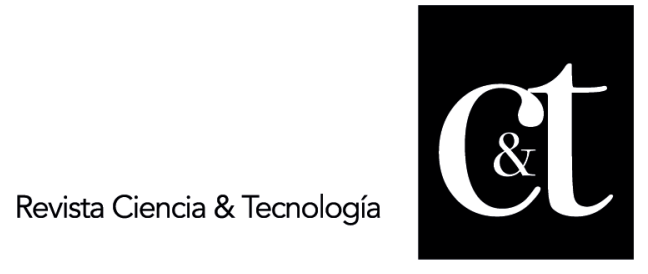

No. 33, 31 de enero de 2022

ISSN impreso: 1390 - 6321

ISSN online: 2661 - 6734

El Marketing tiene que estar orientado ahorita al área digital, todo lo que es delivery, la academia que desarrolle ese tipo de alternativas será espectacular. (EF, UniLimpio)

Ahora el Marketing digital se conjuga con el conocimiento de la estrategia de Marketing puesto que se quiere adaptar a una nueva realidad, mucho más tecnológica. Hoy por hoy el Marketing debe ser digital, la comunicación tiene que ser digital, las promociones tienen que ser digitales. Luego, la persona que está encargada de trabajar la parte estratégica, también tiene que trabajar en la parte digital; es actualmente la que tiene mayor demanda. ... Es justamente con la parte del manejo de todo lo que es el SEO, el SEM, el tema del ecommerce es muy importante porque ahora las ventas se están dando en línea, entonces eso no quiere decir que la persona deba tener todas las habilidades en tecnología, pero si debe conocer mucho ¿cómo implementar o cómo ir implementando su estrategia a la parte del Marketing digital. El tema del Marketing digital vino para quedarse; esa es una realidad. (SF, Kotra).

Barragán de la Parra (2021) sostiene que la multidisciplinariedad de la mercadotecnia le permite al profesional desempeñarse en diversas áreas de una firma: (1) investigar el mercado para identificar los beneficios que el cliente necesita o desea; (2) desarrollar bienes y servicios que respondan a la demanda del mercado; (3) dotar a las marcas de personalidad para lograr un posicionamiento comercial; (4) planear estrategias de precio, plaza y comunicación, establecer relaciones valiosas con el cliente, generar identidad y vinculación con las empresas; (5) promover a través de las acciones sociales, gerenciales y comerciales el bienestar del consumidor y el equilibrio ecológico de la sociedad a la que pertenece.

\section{Pregunta 3}

En la carrera de Marketing, ¿qué asignaturas son aquellas en las que la academia debe hacer más énfasis o las más importantes?

Al consultar sobre las asignaturas más importantes a dictarse en la carrera de Mercadotecnia, los empresarios y directivos consultados coinciden en que las materias NeuroMarketing, Marketing digital, Investigación de Mercados en ambientes digitales, Dirección de Marketing -que involucre el desarrollo de productos y marcas-, Comportamiento del consumidor, Publicidad creativa, Administración de Empresas y Emprendimiento deben tener la prioridad del caso.

A las materias tradicionales le añadiría la Publicidad creativa porque eso deja despertar tu imaginación y sabemos que en Marketing debemos tener imaginación para crear, muchas veces se crea de la nada, es como en este momento también está en boga "véndele a la mente, no a la gente". Por otra parte, el Neuromarketing, también va a ayudar a que el alumno retraído despierte, porque todos los seres humanos necesitamos un estímulo para despertarnos y sentirnos vivos. (MS, Solís Pharma).

Veo que algunas podrían integrarse unas con otras, ejemplo: Investigación de Mercados o Investigación de Mercados en ambientes digitales podría ser una sola. 




No. 33, 31 de enero de 2022

ISSN impreso: 1390 - 6321

ISSN online: 2661 - 6734

La Investigación de Mercado se nutre de todos los datos que te pueda estar dando Google Analytics ... es una investigación que te sirve para generar estrategias.

Dirección de Marketing es muy importante, porque aquí debemos abordar lo que es la planificación y con la planificación están las proyecciones. Diría que la parte de Dirección de Marketing y de Dirección Comercial tiene que dictarse en una sola asignatura.

Súper importante la parte de Emprendimiento, ahora más que nunca hay que conocer ¿cómo ha cambiado el nuevo sistema de economías y cómo generar fuentes de empleo?

Es también necesaria la Administración de Empresas, porque cubre lo contable y financiero. Es importante que el marquetero sepa leer estados financieros. En la parte de Dirección de Marketing puede entrar la parte de desarrollo de productos y marcas. (SF, Kotra).

Fuera de lo tradicional creo que lo imprescindible al momento es el Marketing Estratégico, Marketing Digital, Comportamiento del Consumidor, Investigación de Mercados, en ámbitos digitales y el tema de Emprendimiento,... Investigación de Mercados es radical. Usualmente lo que uno hace es tercerizar la Investigación de Mercados por lo se necesita un que interprete esa investigación para generar la estrategia comercial, pero sería mejor que en la empresa pueda hacerse la investigación y el diseño de estrategias. (CP, PremiunCorp)

En vista de que la mercadotecnia no es una ciencia sino una disciplina, se alimenta de diferentes áreas de estudio como la psicología, sociología, las finanzas y la antropología; también tiene injerencia con la comunicación, la administración y el comercio e incluso se entrevera con algunas áreas de la medicina, en lo que se refiere a la neurociencia. Está a la vanguardia de la tecnología a través de la cual se diseña y desarrolla todo el comercio electrónico, las redes sociales y tanto las herramientas como las acciones y mediciones. (Barragán de la Parra, 2021)

\section{Pregunta 4}

¿Cuál es el perfil de un profesional de Marketing que usted contrataría?

A esta interrogante en términos generales los consultados concluyen que es importante que el candidato tenga aptitud y actitud, que son palabras casi homófonas pero que son distintas y en sí se complementan. Se solicita una persona con las destrezas y habilidades anteriormente mencionadas pero también con una actitud proactiva, de empoderamiento, de innovación, de ir más allá; de visión holística y de muchas ganas para salir adelante.

Una persona que no sepa expresarse, que no sepa escribir y que no pueda hacer un buen análisis numérico, es una persona que en realidad me va a dar muchísimos problemas. Por otro lado contrataría a una persona que tenga ganas de comerse el mundo, que venga con nuevas ideas, que piense más allá afuera de la caja, esa persona que tenga esa visión helicóptero, que no mire el árbol, sino que mire el bosque. ( $A Z, U D L A)$. 


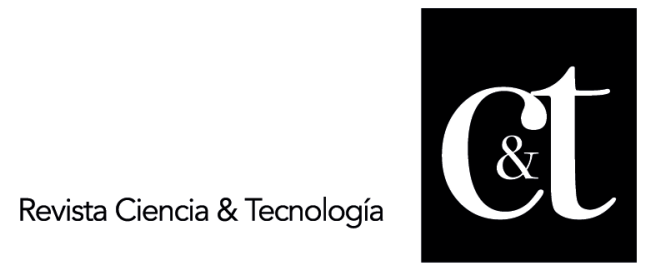

No. 33, 31 de enero de 2022

ISSN impreso: 1390 - 6321

ISSN online: 2661 - 6734

Yo consideraría las destrezas, una persona que sea analítica y creativa. Es también importante la actitud también, todo el mundo habla de una actitud, pero sí la persona de Marketing debe tener una actitud, a ser propositiva, a encontrar nuevas maneras de trabajar, tener un pensamiento lateral, tener esa curiosidad por el consumidor, tener esa curiosidad de hacer bien las cosas y trabajar en pos de un cliente que tiene que estar satisfecho; entonces, pues si tiene esas cualidades pues sería contratado. (SF, Kotra).

Las personas de Marketing tienen que ser poli funcionales, holísticos a la par con el perfil comercial y con conocimientos de Marketing. Según mi apreciación, las personas del Departamento de Marketing deben tener una mentalidad muy globalizada, debe ser una persona a la cual se le pueda apreciar un buen nivel de cultura general, por lo menos, ya que debe entender las circunstancias que lo rodean muy bien, ser alguien informado y aparte tener una noción de lo que es cultura mundial y poder siempre innovar. La persona de marketing de tener ese perfil de innovador que planifica y crea estrategias al mismo tiempo para luego ejecutarlas. (IJ, HDP Agencia mayorista de turismo).

\section{Pregunta 5}

¿Qué mejoras al programa de estudios de la carrera de Marketing propondría?

Los directivos fueron coincidieron en que la malla de estudios debe considerar la praxis para el estudiante. No estaría por demás que en las aulas se discuta sobre comportamiento del consumidor en el campo físico de expendio, sean centros comerciales, puntos de ventas o incluso una avenida así como casos reales por resolver de distintas organizaciones.

Construiría una malla relacionando negocios con el mundo digital y que pueda terminar al final en un producto con algún tipo de innovación, ejemplo: una App; algo que verdaderamente traduzca lo que decimos. Exigir que dentro de la metodología se incorpore más la práctica en el marketing, que existan materias en las que los profesores acompañen a los estudiantes a diferentes cadenas, visitas a empresas,... como qué ese olor a marketing de la realidad es el que te va un poquito a bajar los pies a la tierra. Es distinto pensar en cómo se vende la lotería la lotería en New York a como se la vende en Daule, en Nobol o en algún pueblito recinto. El trabajo de campo sirve bastante. (RP, Mia Brands Network)

Me pregunto ¿por qué la Universidad de los Zamoranos tiene esta credibilidad? Porque los chicos van y no van con papel y pluma, ni a un pupitre, ni a una aula universitaria, ellos van al campo, y el primer año es una formación en la que tenga que ver factores importantes que tienen que ver con el desarrollo profesional, entonces, para mí Marketing hoy y la mayoría de carreras deben hacer lo que hace la carrera de Medicina a los médicos se los forman, primero hacen visitas, luego hacen las guardias, luego hacen el internado ... por ahí la Universidad tiene que reinventarse. (LP, Mariscos Flipper).

El presupuesto de las empresas se ha ido reduciendo, entonces la recomendación sería que se enfoque más al tema práctico, y se enfoque más a cómo generar la venta y hay un tema más interesante que es el punto de venta, 31

Revista científica Ciencia y Tecnología Vol 22 No 33 págs. 21-36 http://cienciaytecnologia.uteg.edu.ec 


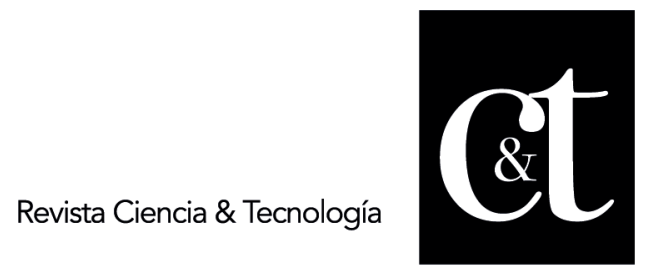

No. 33, 31 de enero de 2022

ISSN impreso: 1390 - 6321

ISSN online: 2661 - 6734

en el punto de venta es todo un arte en cómo desarrollar a las tiendas en el post-COVID. El trabajo es una empresa en constante crecimiento es bajo esquemas de presión que a lo mejor el estudiante no lo vive en las aulas. Existen prestigiosas universidades internacionales que exige sus profesores colaborar en el Directorio de firmas y a resolver casos reales. (AC, Elementia - Eternit).

García \& Echegaray (2018) sostienen que los planes de estudio de la formación universitaria deberían ser reorientados de forma que su nivel de adecuación a la empresa cubriese cualquier déficit. Déficits que por otra parte atañen a campos tan importantes como las habilidades sociales y de comunicación, el marketing digital, o las destrezas para trabajar en organizaciones líquidas

\section{Publicidad de diversas IES}

Con respecto a la publicidad de las IES a nivel de grado y posgrado en la ciudad de Guayaquil, se consideró los días de matriculación a un nuevo semestre en el mes de septiembre de 2020 para utilizar la técnica de la observación. Algunos docentes visitamos físicamente lugares estratégicos, el resto del equipo de investigación visitó los sitios web, navegó en redes sociales y estuvo atento a todo intento publicitario que aparecían en plataformas que brindan correo electrónico gratuito como Google, Yahoo, Microsoft, etc.

IES con mayor cantidad de publicidad

Tabla 1: IES con mayor cantidad de publicidad en la ciudad

\begin{tabular}{lcc}
\hline \multicolumn{1}{c}{ IES } & $\begin{array}{c}\text { Cantidad } \\
\text { de } \\
\text { anuncios }\end{array}$ & Porcentaje \\
\hline $\begin{array}{l}\text { Universidad } \\
\text { Ecotec }\end{array}$ & 19 & $27.9 \%$ \\
\hline UCSG & 12 & $17.6 \%$ \\
\hline UEES & 9 & $13.2 \%$ \\
\hline $\begin{array}{l}\text { Tecnológico } \\
\text { Argos }\end{array}$ & 5 & $7.4 \%$ \\
\hline UIDE & 4 & $5.9 \%$ \\
\hline UTEG & 4 & $5.9 \%$ \\
\hline $\begin{array}{l}\text { Tecnológico } \\
\text { ITB }\end{array}$ & 3 & $4.4 \%$ \\
\hline UTPL & 3 & $4.4 \%$ \\
\hline ESAI Business & 2 & $2.9 \%$ \\
\hline School & 2 & $2.9 \%$ \\
\hline UMET & 1 & $1.5 \%$ \\
\hline ISUF & 1 & $1.5 \%$ \\
\hline UNEMI & 1 & $1.5 \%$ \\
\hline $\begin{array}{l}\text { Universidad } \\
\text { del Río }\end{array}$ & 1 & $1.5 \%$ \\
\hline $\begin{array}{l}\text { Universidad } \\
\text { Indoamérica }\end{array}$ & 1 & $1.5 \%$ \\
\hline UTE & 68 & $100 \%$ \\
\hline TOTAL & & \\
\hline Fuente: Elaboracón propia & \\
\hline
\end{tabular}

Fuente: Elaboración propia 


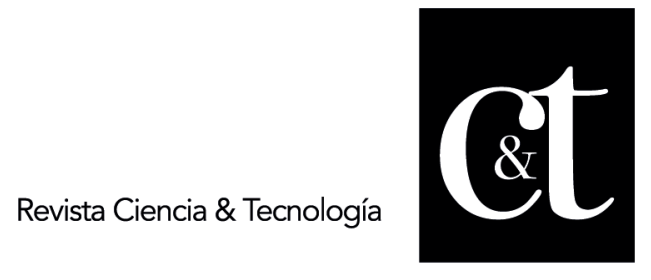

No. 33, 31 de enero de 2022

ISSN impreso: 1390 - 6321

ISSN online: 2661 - 6734

Debe tenerse presente que estos resultados son referenciales pues obedecen a las fechas indicadas y conforme transcurre el tiempo y la aparición de nuevas ofertas por parte de la competencia; algunas IES agregan más publicidad y otras no, según lo observamos. Entre los resultados destacamos que las IES que más publicidad realizan de manera física y digital según lo observado durante 45 días fueron: Universidad Ecotec, UCSG, UEES, Tecnológico Argos, UIDE, etc con un $27.9 \%, 17.6 \%, 13.2 \%, 7.4 \%, 5.9 \%$ con el porcentaje detallado en un total de 68 observaciones. Lo expuesto se ilustra en la Tabla 1.

\section{Medios y lugares elegidos por las IES para presentar publicidad}

Los lugares si bien se veían ingeniosos, eran al mismo tiempo estratégicos. A nivel físico las IES consideraron: (1) avenidas principales, vallas publicitarias en ciertas calles, el aeropuerto externa e internamente, paredes de ciertas construcciones ubicadas en avenidas suburbanas; (2) displays implementados en centros comerciales y aquí recalcamos que en ciertos displays se presentaba propaganda incluso de 2 o más IES en el mismo dispositivo; (3) no se les escapó a las IES considerar también los cines y los baños de los cines en centros comerciales; (4) parabrisas de taxis y buses.

A nivel digital (1) no podían faltar las redes sociales -WhatsApp, Facebook, Instagram, Lindedln, Twitter, Youtube-, ..; (2) plataformas de búsqueda: Google, Bing, etc; (3) Ecuavisa on line; (4) espacios publicitarios en noticieros de TV, plataformas de correos electrónicos gratuitos -Google, Yahoo, Microsoft, etc.-; (5) sin olvidarse de Direct TV y los medios de comunicación tradicionales: TV, radio y prensa escrita.

Según lo encontrado se muestra un conteo de las observaciones realizadas en la tabla 2.

Tabla 2: Medios de publicidad empleados por las IES

\begin{tabular}{lcc}
\hline \multicolumn{1}{c}{$\begin{array}{l}\text { Medio de } \\
\text { publicidad }\end{array}$} & $\begin{array}{c}\text { Cantidad } \\
\text { de } \\
\text { anuncios }\end{array}$ & Porcentaje \\
\hline $\begin{array}{l}\text { Vallas } \\
\text { publicitarias }\end{array}$ & 12 & $17.6 \%$ \\
\hline $\begin{array}{l}\text { Publicidad en } \\
\text { TV }\end{array}$ & 9 & $13.2 \%$ \\
\hline $\begin{array}{l}\text { Publicidad en } \\
\text { Google }\end{array}$ & 4 & $5.9 \%$ \\
\hline $\begin{array}{l}\text { Redes sociales: } \\
\text { WhatsApp }\end{array}$ & 3 & $4.4 \%$ \\
\hline $\begin{array}{l}\text { Publicidad en } \\
\text { buses }\end{array}$ & 3 & $4.4 \%$ \\
\hline $\begin{array}{l}\text { Redes sociales: } \\
\text { Facebook }\end{array}$ & 1 & $1.5 \%$ \\
\hline $\begin{array}{l}\text { Redes sociales: } \\
\text { Instagram }\end{array}$ & 1 & $1.5 \%$ \\
\hline $\begin{array}{l}\text { Redes sociales: } \\
\text { Twitter }\end{array}$ & 1 & $1.5 \%$ \\
\hline $\begin{array}{l}\text { Otros medios } \\
\text { publicitarios }\end{array}$ & 34 & $50 \%$ \\
\hline $\begin{array}{l}\text { TOTAL } \\
\text { Fuente: Elaboración propia }\end{array}$ & 68 & $100 \%$ \\
\hline
\end{tabular}




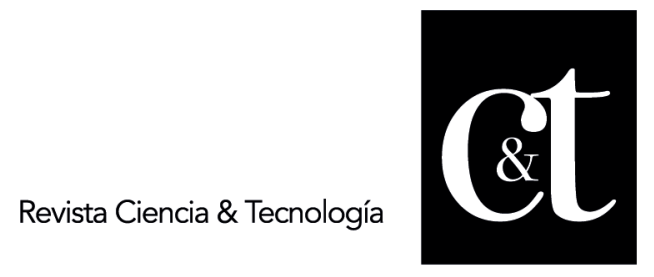

No. 33, 31 de enero de 2022

ISSN impreso: 1390 - 6321

ISSN online: 2661 - 6734

\section{Variables principales presentadas en la redacción publicitaria}

Si bien es cierto en cuanto a oferta académica de una IES con respecto una carrera se pueden considerar varias decenas de variables. Al ser los mensajes de publicidad cortos y objetivos las variables comunes que las IES quieren que el consumidor tenga presente son: título que otorga, duración, precio, modalidad de estudio, horario, convenios, contenidos promocionales. Aunque al acercarse a las oficinas o realizar un contacto telefónico pueden también brindarnos información sobre: número de materias, formas de pago \& financiamiento, becas y ayudas, tiempo de la IES en el mercado, facilidad de transportación que brinda la IES, profesores principales, pasantías y/o prácticas pre-profesionales, tecnología que brinda, infraestructura, curso introductorio, entre otras.

\section{Conclusiones}

\section{Requerimientos del mercado laboral}

La educación superior en general, exige una revolución profunda en nuestro sistema educativo a todo nivel. Es imprescindible que la práctica se ofrezca a la par con los conocimientos teóricos para lo cual se debe contar con el mejor talento humano posible y respaldar la investigación científica dotando al claustro docente y a los estudiantes de los recursos necesarios para llevarla a cabo.

El mercado laboral demanda muchas cualidades en un profesional del Marketing que deben ser desarrolladas debidamente por la academia; estos deben ser personas de conocimiento sólidos en lo técnico, que exploten el uso de la tecnología: piensen en apps, programas, plataformas, algoritmos, interacciones con una vasta cultura técnica y general.

\section{Publicidad de diversas IES}

La publicidad ha penetrado fuertemente en educación superior privada de las universidades se concentra en la ciudad de Guayaquil principalmente en los meses de abril y septiembre a nivel de grado y trimestralmente a nivel de posgrado.

El gasto en publicidad es bastante variado o tal vez desigual a nivel de ciertas IES. Algunas por captar clientes potenciales prometen mucho, lo importante es que esto se de en el transcurrir de la carrera del estudiante.

Con las variables mencionadas (título que otorga, duración, precio, modalidad de estudio, horario, convenios, contenidos promocionales, etc.) en este estudio se pretende cubrir los factores críticos que influyen en que los bachilleres, estudiantes y graduados elijan entre una u otra universidad. Se destaca que como parte de una hábil, estratégica y creativa redacción publicitaria se incluyen comúnmente en los anuncios los contenidos promocionales.

Futuras investigaciones podrían tratar sobre una evaluación de la publicidad en línea de los centros de estudio en el Ecuador tomando en cuenta opiniones de expertos y potenciales demandantes. 


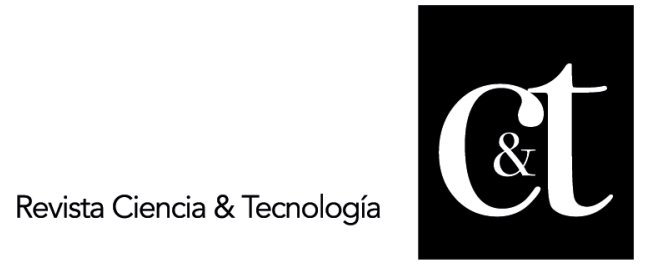

No. 33, 31 de enero de 2022

ISSN impreso: 1390 - 6321

ISSN online: 2661 - 6734

\section{Referencias bibliográficas}

Adecco. (2021). Obtenido de Adecco Ecuador: https://www.adecco.com.ec/

Arias, F. (2012). El proyecto de investigación. Introducción a la metodología científica. Caracas: Episteme.

Barbosa, J., Barbosa, J., \& Rodríguez, M. (2015). Concepto, enfoque y justificación de la sistematización de experiencias educativas. Una mirada desde y para el contexto de la formación universitaria. Perfiles educativos, 130-149.

Obtenido

de http://www.scielo.org.mx/pdf/peredu/v37n149/v37n149a8.pdf

Barragán de la Parra, R. (2021). El desafío del profesional en mercadotecnia. Círculo de escritores. Obtenido de https://web.mediasolutions.mx/Notas/?id=202105271442344462

Begoña, M. (2004). Educación y nuevas tecnologías. Educación a distancia y educación virtual. Teoría y didáctica de las Ciencias Sociales, 209-222.

Bernal, C. (2010). Metodología de la investigación. Administración, Economía, Humanidades y Ciencias Sociales. Bogotá: Prentice Hall.

Caetano, G. (2003). Marketing en los servicios de educación: Modelos de percepción de calidad. España.

Cárcamo, H. (2005). Hermenéutica y análisis cualitativo. Cinta moebio, 13.

Carrillo, M., \& Rodriguez, A. (2018). El ecosistema programático. La nueva publicidad digital que conecta datos con personas. El profesional de la información, 195-201.

Cuáles deben ser las competencias y las habilidades del profesional del marketing?. Una visión exploratoria desde la perspectiva profesional y académica. (2018). Esic Market Economics and Business Journal, 261-283. Obtenido

de https://www.esic.edu/documentos/revistas/esicmk/1536922661_E.pdf

Duro, V., \& Gilart, V. (2016). La competitividad en las Instituciones de Educación Superior: aplicación de filosofías de gestión empresarial. Gestión del proceso de negocio. Economía y Desarrollo.

Fresno, C. (2019). Metodología de la Investigación. Córdoba: El Cid Editor.

Gómez, S. (2012). Metodología de la Investigación. México: Red Tercer Milenio.

Hernández, R., Fernández, C., \& Baptista, M. (2014). Metodología de la investigación. México D.F.: McGraw Hill.

Nacional, A. (2021). Ley Orgánica de Defensa al Consumidor. Ediciones legales. Obtenido de https://www.dpe.gob.ec/wpcontent/dpetransparencia2012/literala/BaseLegalQueRigeLaInstitucion/Ley OrganicadelConsumidor.pdf

Núñez, E. (2010). Los incentivos que motivan a los jóvenes a estudiar formación profesional. Cómo mejorar su horizonte educativo con el Marketing. Prisma social, 1-34.

Rodriguez, E., Pedraja, L., \& Ganga, F. (2017). La relación entre los estilos de liderazgo y el desempeño de los equipos de dirección intermedia: un estudio exploratorio desde Chile. Contabilidad y Negocios, 129-144.

Rosario, J. (2006). TICs, su uso como herramienta para el fortalecimiento y desarrollo de la educación virtual. Obtenido de http://www.cibersociedad.net/archivo/articulo.php?art=221 


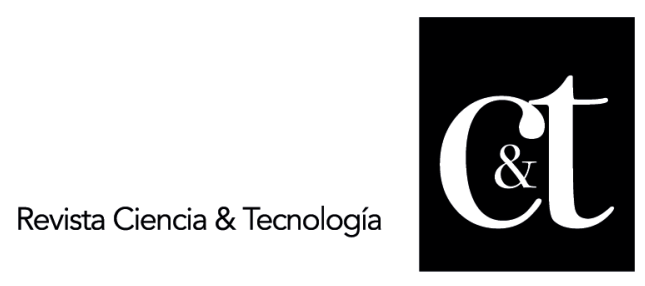

No. 33, 31 de enero de 2022

ISSN impreso: 1390 - 6321

ISSN online: 2661 - 6734

Segura, A., Paniagua, F., \& Moreira, C. (2019). Evaluación de la publicidad de universidades en la prensa escrita de Ecuador, un estudio longitudinal. Obra digital, 109-124.

SENESCYT. (2021). Obtenido de Secretaría de Educación, Ciencia, Tecnología e Innovación: https://www.educacionsuperior.gob.ec/

Zapata, G., \& Tejeda, I. (2016). Publicidad engañosa en Educación Superior: definiciones, regulación y una revisión de la publicidad en prensa escrita. Calidad en la Educación, 197-242. 
No. 33, 31 de enero de 2022

ISSN impreso: 1390 - 6321

ISSN online: 2661 - 6734 
No. 33, 31 de enero de 2022

ISSN impreso: 1390 - 6321

ISSN online: 2661 - 6734 